\title{
Accurate Estimation of the Fisher Information Matrix for the PET Image Reconstruction Problem
}

\author{
Quanzheng Li, Student Member, IEEE, Evren Asma, Member, IEEE, Jinyi Qi, Senior Member, IEEE, \\ James R. Bading, and Richard M. Leahy*, Fellow, IEEE
}

\begin{abstract}
The Fisher information matrix (FIM) plays a key role in the analysis and applications of statistical image reconstruction methods based on Poisson data models. The elements of the FIM are a function of the reciprocal of the mean values of sinogram elements. Conventional plug-in FIM estimation methods do not work well at low counts, where the FIM estimate is highly sensitive to the reciprocal mean estimates at individual detector pairs. A generalized error look-up table (GELT) method is developed to estimate the reciprocal of the mean of the sinogram data. This approach is also extended to randoms precorrected data. Based on these techniques, an accurate FIM estimate is obtained for both Poisson and randoms precorrected data. As an application, the new GELT method is used to improve resolution uniformity and achieve near-uniform image resolution in low count situations.
\end{abstract}

Index Terms-Fisher information matrix, image reconstruction, PET, uniform resolution.

\section{INTRODUCTION}

$\mathbf{S}$ TATISTICAL positron emission tomography (PET) image reconstruction methods, such as penalized maximum-likelihood (ML), can produce improved spatial resolution and variance properties over traditional filtered back-projection methods through accurate physical and statistical modeling. In recent years, properties of such implicitly defined estimators have been analyzed and applications based on these analyses have been developed.

Fessler and Rogers [1], [2] used approximations based on the Taylor series expansion of the implicit function relating the observed data to the reconstructed image to obtain closed form approximations for the covariances of reconstructions, as well as the local impulse response (LIR) from which resolution can be inferred. Qi and Leahy [3] extended these results and derived simplified expressions for the LIR, contrast recovery coefficients (CRCs), and variances for each voxel. Stayman and Fessler designed penalties for both spatially invariant [4] and

Manuscript received December 31, 2003; revised June 7, 2004. This work was supported in part by the National Institute of Biomedical Imaging and Bioengineering under Grant R01 EB000363. The Associate Editor responsible for coordinating the review of this paper and recommending its publication was $\mathbf{J}$. A. Fessler. Asterisk indicates corresponding author.

Q. Li and E. Asma are with the Signal and Image Processing Institute, Univ. of Southern California, Los Angeles, CA 90089 USA.

J. Qi is with the Center for Functional Imaging, LBL, University of California, Berkeley, CA 94270 USA.

J. R. Bading is with the PET Imaging Sciences Center, Univ. of Southern California, Los Angeles, CA 90033 USA.

*R. M. Leahy is with the Signal and Image Processing Institute, Univ. of Southern California, Los Angeles, CA 90089 USA (e-mail: leahy@sipi.usc.edu).

Digital Object Identifier 10.1109/TMI.2004.833202 variant [5] systems to achieve near-uniform and isotropic resolution. They also developed a fast method to obtain approximate resolution and covariance expressions for penalized ML estimators in single photon emission computed tomography (SPECT) [6].

Bonetto et al. proposed a fast and accurate approximation for channelized Hotelling observer statistics [7] for computer observers applied to penalized ML reconstructions. Barrett et al. [8] derived figures of merit for image quality based on the performances of computer observers on specific detection and estimation tasks. Kijewski et al. investigated the effects of SPECT collimator sensitivity [9] and system geometry [10] on several estimation tasks. Qi studied lesion detectability [11], and the optimization of PET system design for lesion detection [12], again for the case of penalized ML reconstructions.

The Fisher information matrix (FIM) is not only important in itself, in that it provides a bound on the variances of unbiased image estimates, but also all of the expressions derived in the work mentioned above depend either on the entire FIM or its diagonal elements. The exact FIM is a function of the reciprocal of the measurement means at individual sinogram bins, which are unknown for real datasets. In practical applications, we can compute an estimate of the FIM by substituting some function of the data in place of the true mean values. While the approximation $1 / \bar{y} \approx 1 / y$, where $\bar{y}$ and $y$ denote the mean and observed counts at a sinogram bin, respectively, is commonly used in the above literature and works well for high count studies, it is heavily biased in low count situations. An estimate for the FIM can also be computed by using the forward projection of a preliminary reconstruction as mean data, however, such an estimate has no proven optimality properties. The primary objective of this paper is to optimally estimate the FIM under low count situations.

In practice, most $\mathrm{PET}$ data is precorrected for randoms, which results in a non-Poisson model. Yavuz and Fessler [13] proposed a "shifted Poisson model" to provide an approximation to the log-likelihood function of the exact distribution of randoms precorrected data. This approximate log-likelihood function is widely used in different reconstruction methods [13]-[16]. Here we use the shifted-Poisson model to adapt the GELT method to provide an optimized estimate of the FIM for randoms precorrected data.

This paper is organized as follows: Section II describes the proposed estimator for reciprocal means and the FIM. In Section III, we evaluate the accuracy of these reciprocal mean and FIM estimates under the Poisson and shifted Poisson models. Section IV includes results from a specific application in which 
we demonstrate improvement in resolution uniformity using the new FIM estimation method.

\section{OPTIMAL ESTIMATION OF FISHER INFORMATION}

\section{A. The Fisher Information Matrix}

The FIM, $\mathbf{F}(\mathbf{x})$, provides a bound on the covariance matrix of an unbiased estimator $\hat{\mathbf{x}}$, whose density depends on a set of parameters $\mathbf{x}$, in the sense that $\mathbf{C}_{\hat{\mathbf{x}}}-\mathbf{F}(\mathbf{x})$ is a positive semidefinite matrix. Here $\mathbf{C}_{\hat{\mathbf{x}}}$ denotes the covariance matrix of the estimator and $\mathbf{F}$ denotes the FIM with elements

$$
\begin{aligned}
{[\mathbf{F}]_{i j} } & =-E\left[\frac{\partial \ln p(\mathbf{y} ; \mathbf{x})}{\partial x_{i}} \frac{\partial \ln p(\mathbf{y} ; \mathbf{x})}{\partial x_{j}}\right] \\
& =-E\left[\frac{\partial^{2} \ln p(\mathbf{y} ; \mathbf{x})}{\partial x_{i} \partial x_{j}}\right] .
\end{aligned}
$$

For the problem of estimating an image $\mathbf{x}$ from Poisson data $\mathbf{y}$ with mean $\mathbf{P x}$, the FIM is given by

$$
\mathbf{F}=\mathbf{P}^{T} D\left\{\frac{1}{\bar{y}_{i}}\right\} \mathbf{P}
$$

where $\mathbf{P}$ is the detection probability matrix, $D\{\cdot\}$ denotes a diagonal matrix, the bar denotes the mean, and we assume that $\bar{y}_{i}>0 \forall i$.

When $\mathbf{F}$ is estimated from measured sinogram data, the accuracy of the estimate will depend on the accuracy of the reciprocal mean estimates at individual sinogram bins (i.e., the $1 / \bar{y}_{i}$ terms). The "direct plug-in" (DPI) method proposed in [1] uses $1 / \max \left(y_{i}, \epsilon\right)$ where $\epsilon>0$ ensures the positivity of the denominator when $y_{i}=0$. A simple but quite effective modification, which we refer to as the "modified plug-in" (MPI), is the estimator $1 /\left(y_{i}+1\right)$ [17]. These two estimators lead to the following FIM approximations:

$$
\begin{aligned}
\hat{\mathbf{F}}_{\mathrm{DPI}} & =\mathbf{P}^{T} \mathbf{D}\left\{\frac{1}{\max \left(y_{i}, \epsilon\right)}\right\} \mathbf{P} \\
\hat{\mathbf{F}}_{\mathrm{MPI}} & =\mathbf{P}^{T} \mathbf{D}\left\{\frac{1}{y_{i}+1}\right\} \mathbf{P}
\end{aligned}
$$

where $\mathbf{D}\{\cdot\}$ denotes a diagonal matrix. Fig. 1 shows the relative biases (i.e., $E(\hat{\theta}) / \theta$ where $\theta=1 / \bar{y}$ is the estimated parameter) of the DPI and MPI methods applied to a single Poisson random variable as a function of the mean activity $\bar{y}$. Both methods work very well at high levels of activity, producing almost unbiased estimates with low variance for reciprocal means, which in turn results in accurate FIM estimates. However, both methods perform poorly in low count studies, especially when the average number of counts per sinogram bin falls below 5 . This poor performance is primarily due to the large bias in reciprocal mean estimates which will result in inaccurate FIM estimates.

These methods also provide large variance $1 / \bar{y}_{i}$ estimates at low counts compared to high counts, however, this effect is ameliorated in the FIM estimates due to the averaging caused by premultiplication by $\mathbf{P}^{T}$ and post-multiplication by $\mathbf{P}$. This observation also forms the basis of the FIM estimation technique presented in this paper. In particular, relatively high variance reciprocal mean estimates are allowable due to this averaging effect. On the other hand, bias in the reciprocal mean estimates is undesirable, since consistent positive or negative bias on the

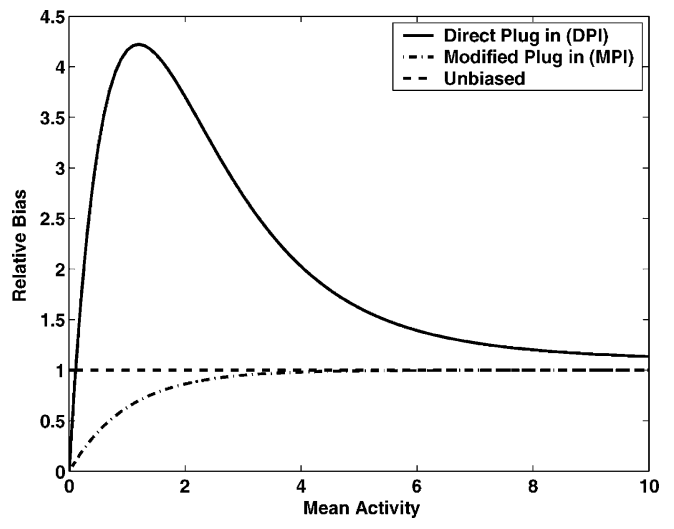

Fig. 1. The relative bias of DPI and MPI methods versus mean activity $\bar{y}$. The relative bias is computed as $E(\hat{\theta} / \theta)$ where $\hat{\theta}$ is the estimator of $\theta=1 / \bar{y}$.

estimate $1 / \bar{y}$ results in a correspondingly biased FIM estimate. Therefore, we trade off variance in exchange for low bias in estimating reciprocal means, and the resulting FIM estimate outperforms both DPI and MPI at all activity levels.

\section{B. FIM Estimation Model}

Suppose $y$ is a realization of a Poisson random variable with mean $\bar{y}$. The parameter of interest $\theta$ and the form of the estimator $\hat{\theta}$ that we use is as follows:

$$
\theta=\frac{1}{\bar{y}} \quad \hat{\theta}=\frac{D_{y}}{y+1}
$$

where $D_{y}$ is a sequence of constants defined for integer values of $y$ as

$$
D_{y}=\left\{\begin{array}{ll}
C_{y}, & \text { for } y \leq N \\
1, & \text { for } y>N
\end{array} .\right.
$$

Note that the $N$ constants, $C_{1}, \ldots, C_{N}$, which appear in (6), define the estimator and are the coefficients that we need to determine. This form is a generalization of the MPI method which is the special case $C_{1}=\cdots=C_{N}=1$. In all of the results presented in this paper, $N=5$ was chosen since we observed little additional improvement for $N>5$.

\section{Generalized Error}

Let $\hat{\phi}(\mathbf{z})$ be an estimator of the quantity $\phi$ given observation $\mathbf{z}$. The mean squared error (MSE) is defined as

$$
M=E\left(E\left[(\hat{\phi}(\mathbf{z})-\phi)^{2}\right] \mid \phi\right)=V+B
$$

where $V$ and $B$ are, respectively, the variance and squared bias of the estimator

$$
\begin{aligned}
V & =E\left(E(\hat{\phi}(\mathbf{z})-E(\hat{\phi}(\mathbf{z})))^{2} \mid \phi\right) \\
B & =E\left((E(\hat{\phi}(\mathbf{z})-\phi))^{2} \mid \phi\right) .
\end{aligned}
$$

Our goal here is to accurately estimate the FIM = $\mathbf{P}^{T} \mathbf{D}\left\{1 / \bar{y}_{i}\right\} \mathbf{P}$. The number of terms $n_{i}$ contributing to each diagonal element $\kappa_{j}$ of the FIM is equal to the number of nonzero elements in each column of the sparse matrix $\mathbf{P}$. Consequently, the FIM is less sensitive to standard deviation than bias in the estimates of $1 / \bar{y}_{i}$ by a factor of approximately $\sqrt{n_{i}}$. This observation leads us to define a generalized error, $\mathrm{G}$, 


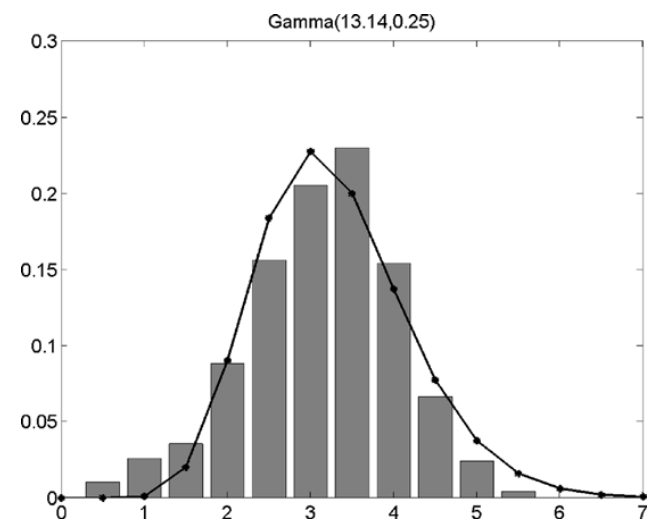

Fig. 2. Least squares fit of the gamma distribution $(13.14,0.25)$ to the histogram of the nonzero entries of the mean value of a sinogram with a total mean activity of $150 \mathrm{~K}$ counts.

that attaches more weight to squared bias than to variance in the estimation of $1 / \bar{y}_{i}$

$$
G \equiv B+w V
$$

where $0 \leq w \ll 1$. Based on the observation above, in practice we choose a value of $w$ equal to the reciprocal of the average number of lines of response that intersect each voxel.

\section{Cost Function}

To compute the generalized error in (10) we need to specify a prior distribution on $1 / \bar{y}$. We assume an independent identically distributed gamma distribution for the mean values of the elements of the sinogram with parameters $\alpha$ and $\beta$

$$
p_{\bar{y}}(x)=\frac{\left(\frac{x}{\alpha}\right)^{\beta-1} e^{-x / \alpha}}{\alpha \Gamma(\beta)} .
$$

Shown in Fig. 2 is a fit of the gamma distribution to the nonzero entries of a noiseless sinogram with a mean of $150 \mathrm{~K}$ counts. The gamma distribution provides a reasonable fit to realistic sinogram histograms, and has the added attraction that it is the conjugate prior for the Poisson data model. With this prior distribution for $\bar{y}$, a change of variables $\theta=1 / \bar{y}$ gives us the prior distribution of $1 / \bar{y}$ as

$$
p_{1 / \bar{y}}(z)=\frac{\left(\frac{1}{\alpha z}\right)^{\beta-1} e^{-1 / \alpha z}}{\alpha \Gamma(\beta)} \frac{1}{z^{2}} .
$$

For each scan, we determine $\alpha$ and $\beta$ by fitting the gamma distribution to the empirical distribution of the data. With a prior distribution available for the parameter of interest, we now define our cost function as the generalized error

$$
\begin{aligned}
G= & G(\mathbf{c})=G\left(C_{0}, \ldots, C_{N}\right) \\
= & \int_{0}^{\infty}\left\{(1-w)\left[\sum_{y=0}^{\infty} \frac{D_{y}}{1+y} \frac{\bar{y}^{y} e^{-\bar{y}}}{y !}-\frac{1}{\bar{y}}\right]^{2}\right. \\
& \left.\quad+w\left[\sum_{y=0}^{\infty}\left(\frac{D_{y}}{1+y}-\frac{1}{\bar{y}}\right)^{2} \frac{\bar{y}^{y} e^{-\bar{y}}}{y !}\right]\right\} \\
& \times \frac{\left(\frac{\bar{y}}{\alpha}\right)^{\beta-1} e^{-\bar{y} / \alpha}}{\alpha \Gamma(\beta)} d \bar{y}
\end{aligned}
$$

where we use the fact that, from (7) and (10), we can write $G=$ $(1-w) B+w M$. Note that $\left\{D_{y}\right\}_{y=0}^{N}$ are related to $\left\{C_{y}\right\}_{y=0}^{N}$ by (6).

By inspection, this cost function can be written in the standard quadratic form as $T(\mathbf{c})=1 / 2 \mathbf{c}^{T} \mathbf{Q} \mathbf{c}+\mathbf{b}^{T} \mathbf{c}+d$. We also note that although infinite summations have to be approximated to compute $\mathbf{b}$, the $\mathbf{Q}$ matrix is a function of only the first $N$ terms in the summations and, hence, can be computed exactly. In addition to the generalized error being a quadratic function of $\mathbf{c}$, the Hessian matrix $\mathbf{Q}$ is also positive definite so that the function is convex and the optimal solution is unique. Since the elements of $\mathbf{c}^{*}=\arg \max _{\mathbf{c} \in \mathbf{R}^{N}} G(\mathbf{c})$ form a look up table, we refer to this method as the "generalized error lookup table" (GELT) method and our FIM estimator becomes

$$
\hat{\mathbf{F}}_{\mathrm{GELT}}=\mathbf{P}^{T} D\left\{\frac{D_{y_{i}}^{*}}{y_{i}+1}\right\} \mathbf{P}
$$

where

$$
D_{y_{i}}^{*}=\left\{\begin{array}{ll}
C_{y_{i}}^{*}, & \text { for } y_{i} \leq N \\
1, & \text { for } y_{i}>N
\end{array} .\right.
$$

We note that it may be desirable to compute the integral in (13) with a lower limit of $\bar{y}=y_{0}>0$. While this ignores errors for $0 \leq \bar{y}<y_{0}$, it allows greater control of errors for $\bar{y} \geq y_{0}$ and also avoids the singularity at $\bar{y}=0$ when computing the integral numerically. We return to this issue in Section III. We also note that although the integral in (13) can be evaluated analytically for special cases such as $\beta=3$ and the integration taken over its full range of zero to infinity, in general it has to be evaluated numerically.

\section{E. Randoms Precorrected Data}

PET data is often precorrected for accidental coincidences using delayed windows. In this case, the measurements are no longer Poisson. To extend the method described above to randoms precorrected data, we combine our FIM estimation technique with the shifted Poisson model proposed by Yavuz and Fessler [13], in which $y+2 \bar{r}$, where $\bar{r}$ is the mean of the randoms, is approximated by a random variable with mean and variance equal to $\bar{y}+2 \bar{r}$. To apply the shifted Poisson model, an estimate of the mean randoms rate for each sinogram element is required. In practice, this can usually be estimated from detector block singles rate information typically included in the header of the sinogram data file. In the simulation results presented in this paper, we used the exact mean random rates.

The FIM resulting from the shifted Poisson model log-likelihood is

$$
\mathbf{F}_{S P}=\mathbf{P}^{T} D\left\{\frac{1}{\bar{y}_{i}+2 \bar{r}_{i}}\right\} \mathbf{P}
$$

and, therefore, the parameter of interest $\theta$ and the form of the estimator $\hat{\theta}$ become

$$
\theta=\frac{1}{\bar{y}+2 \bar{r}} \quad \hat{\theta}=\frac{D_{y}(\bar{r})}{y+2 \bar{r}+1} .
$$

Squared bias and mean squared error are then given by

$$
B=\int_{0}^{\infty}(E(\hat{\theta}-\theta))^{2} p_{\theta}(\theta \mid \bar{r}) d \theta
$$




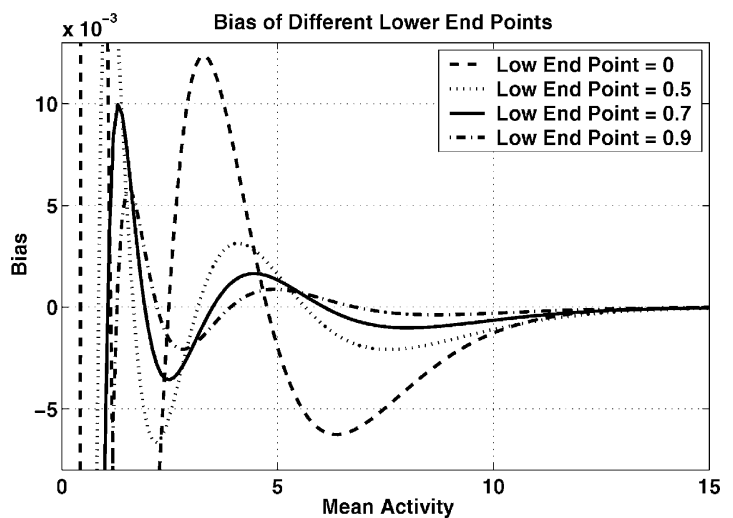

Fig. 3. Bias of different starting points using GELT $(w=1 / 11904, \alpha=5$, $\beta=2$ ) in estimating $1 / \bar{y}_{i}$.

where

$(E(\hat{\theta}-\theta))^{2}=\left[\sum_{y=\lfloor-2 \bar{r}\rfloor}^{\infty} \frac{D_{y}(\bar{r})}{y+2 \bar{r}+1} p_{y+2 \bar{r}}(y+2 \bar{r})-\frac{1}{\bar{y}+2 \bar{r}}\right]^{2}$

here $\lfloor-2 \bar{r}\rfloor$ denotes the smallest integer greater than $-2 \bar{r}$, and

$$
M=\int_{0}^{\infty} E\left(\left[(\hat{\theta}-\theta)^{2}\right]\right) p_{\theta}(\theta \mid \bar{r}) d \theta
$$

where

$E\left(\left[(\hat{\theta}-\theta)^{2}\right]\right)=\sum_{y=\lfloor-2 \bar{r}\rfloor}^{\infty}\left(\frac{D_{y}(\bar{r})}{y+2 \bar{r}+1}-\frac{1}{\bar{y}+2 \bar{r}}\right)^{2} p_{y+2 \bar{r}}(y+2 \bar{r})$.

This leads to the cost function (22) shown at the bottom of the page, which unlike the previous formulation, now includes a dependence on the mean randoms rate, $\overline{\mathbf{r}}$.

From (22) it can be seen that $p(\bar{y} \mid \bar{r})$ is necessary to compute this new cost function. As with the ordinary Poisson model, we fit a gamma distribution to an empirical histogram of the data, in this case, after it has been corrected for randoms. We used an empirical approximation to the exact density for the difference of two Poisson random variables [13] in computing $p_{y+2 \bar{r}}(y+$ $2 \bar{r}$ ) (i.e., we generated pairs of Poisson random variables and took the histogram of their differences). This objective function (22) is again convex and quadratic. Similarly to the case for the Poisson model in Section II-D, the lower limit of the integral in (22) can be larger than $\lfloor-2 \bar{r}\rfloor$ to improve the performance for mean values larger than this limit.

We note that although the GELT method is presented here for a fixed value of $\bar{r}$, it is applicable to the general case where the $\bar{r}_{i}$ 's are different. For each $\bar{r}_{i}=\bar{r}$, we fit the gamma distribution only to the entries of sinogram bins that satisfy $\bar{r}_{i}=\bar{r}$. As a result, $\alpha$ and $\beta$ become functions of $\bar{r}$ and by repeating this

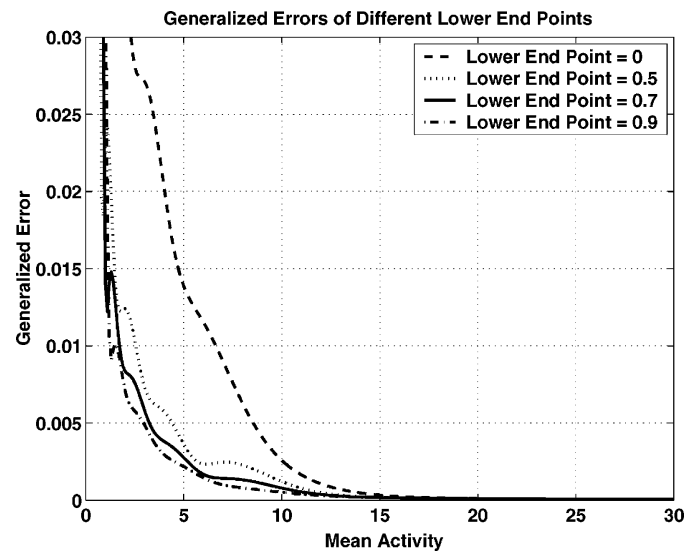

Fig. 4. Generalized errors of different starting points in using GELT ( $w=$ $1 / 11904, \alpha=5, \beta=2$ ) for the estimation of $1 / \bar{y}_{i}$.

process over a range of possible $\bar{r}$ values, we form the entries of our look-up table, which is now two-dimensional (2-D), i.e., a function of both $y$ and $\bar{r}$.

We also note that the DPI and MPI methods can simply be modified to use $1 /(y+2 \bar{r})$ and $1 /(y+2 \bar{r}+1)$, respectively, for estimating $\theta$ under the shifted Poisson model and we use these two forms for comparison in the following.

\section{ESTIMATION ACCURACIES}

\section{A. Choice of Starting Point}

We studied the effect of lowering the lower limit of the integration for the Poisson approximation in Section II-C and the results are shown in Figs. 3 and 4 for a one-dimensional (1-D) Poisson random variable whose mean ranges from 0 to 15 . As the lower limit increases, bias and generalized error after the starting point improve, while performance prior to the starting point deteriorates. Based on the results in Figs. 3 and 4, we chose 0.7 as our integration starting point for the remainder of the studies in this paper. In all of the results presented in this paper, we chose $w=1 /(192 \times 31 \times 2)=1 / 11904$, which is approximately the reciprocal of the average number of detector pairs to which each voxel contributes in the small animal scanner that we simulated.

\section{B. Accuracies of $1 / \bar{y}$ Estimates for Poisson Data}

The bias and variances were compared for the DPI, MPI, and GELT methods and the results are shown in Figs. 5 and 6. For mean values $>20$, all three methods perform almost identically. This also explains why the use of the simple DPI method works well for high count studies. When mean activity falls below 20, the methods exhibit markedly different behavior. As activity drops below 5, the bias of the GELT becomes significantly lower

$$
\begin{aligned}
& G(\mathbf{c} \mid \overline{\mathbf{r}})=G\left(C_{1}, \ldots, C_{N} \mid \overline{\mathbf{r}}\right) \\
& =\int_{\lfloor-2 \bar{r}\rfloor}^{\infty}\left[(1-w)\left[\sum_{y=\lfloor-2 \bar{r}\rfloor}^{\infty} \frac{D_{y}(\bar{r})}{1+y+2 \bar{r}} p_{\bar{y}+2 \bar{r}}(y+2 \bar{r})-\frac{1}{\bar{y}+2 \bar{r}}\right]^{2}+w\left[\sum_{y=\lfloor-2 \bar{r}\rfloor}^{\infty}\left(\frac{D_{y}(\bar{r})}{1+y+2 \bar{r}}-\frac{1}{\bar{y}+2 \bar{r}}\right)^{2} p_{\bar{y}+2 \bar{r}}(y+2 \bar{r})\right] p(\bar{y} \mid \bar{r}) d \bar{y}\right.
\end{aligned}
$$




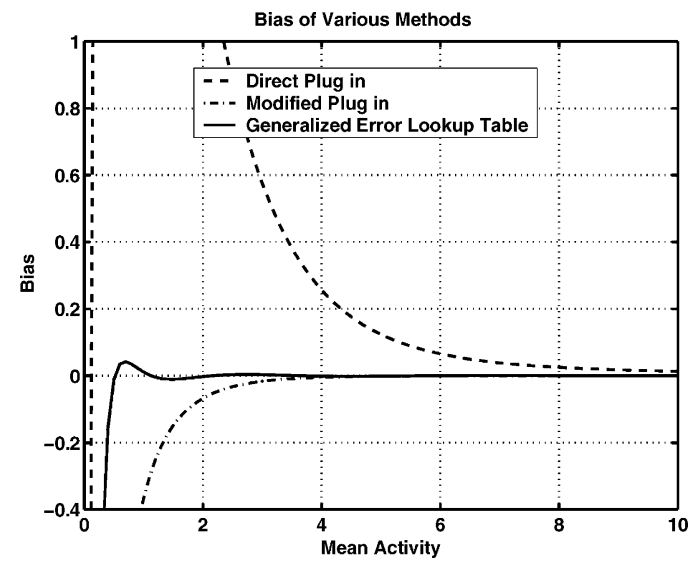

Fig. 5. Bias of $1 / \bar{y}_{i}$ estimates for the DPI, MPI, and GELT methods. The parameters of the gamma distribution in the GELT method were $\alpha=5$, and $\beta=2$.

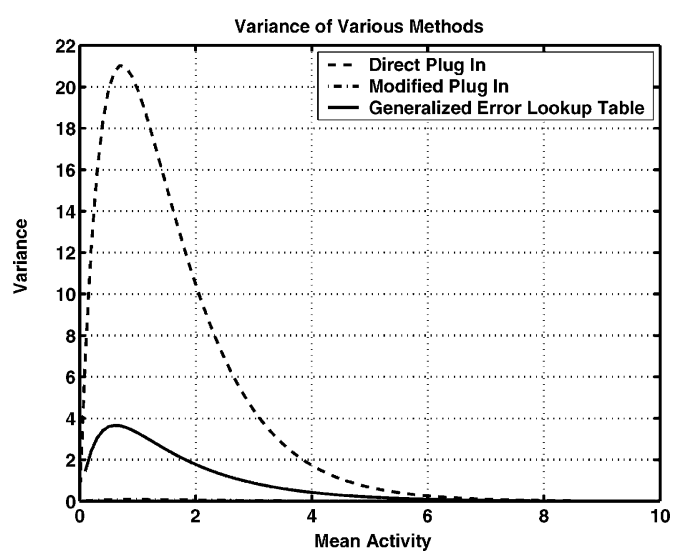

Fig. 6. Variance of $1 / \bar{y}_{i}$ estimates for the DPI, MPI, and GELT methods. The parameters of the gamma distribution in the GELT method were $\alpha=5$, and $\beta=2$.

than that of the MPI, which in turn is far lower than that of the DPI.

From the variance curves in Fig. 6 we see that the variance of the GELT method is larger compared to other methods, which is consistent with its design. This bias-variance trade off works to our advantage since the FIM estimates are a weighted combination of the $1 / \bar{y}_{i}$ estimates and the variance is reduced through this averaging process.

\section{Accuracies of FIM Estimates for Poisson Data}

We simulated a simple single ring scanner, which had the same parameters as the ECAT HR+ (CTI PET Systems, Knoxville, TN). A single slice from the Hoffman brain phantom [18] shown in Fig. 7 was used in our simulation studies. This 2-D simulation configuration was used throughout the simulations presented in the paper.

Monte Carlo simulations were performed to compare the estimates of the diagonal FIM entries $\kappa_{j} \equiv[\mathbf{F}]_{j j}$ computed with different estimation methods. Five levels of low activity were simulated in which average counts per sinogram bin were 1,2,3, 4 , and 5. For each level of activity, 50 noisy sinograms were simulated according to the simulation setup described above. The $\kappa$

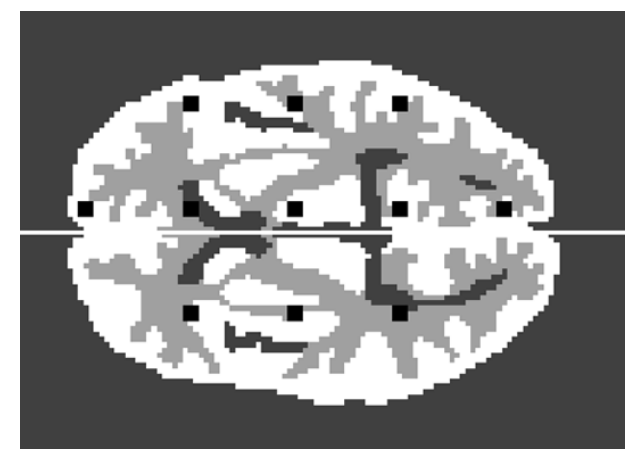

Fig. 7. All simulated sinogram data in this paper were based on this 2-D slice from the 3D Hoffman brain phantom. The white line indicates the 1-D profile through the image at which the FIM or $\kappa$ values are compared in Figs. 8, 9, 12 , and 13. Also shown are 11 points (indicated by small black squares) at which the local resolution was measured for the resolution uniformity study in Section IV-B.

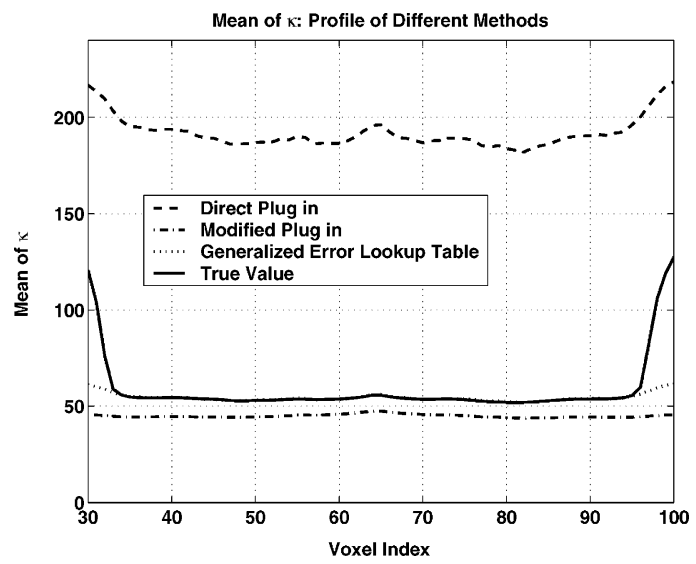

Fig. 8. Profiles of the mean of the $\kappa$ estimates along a line through the center of the field of view of the scanner shown by the white line in Fig. 7. The mean was computed in each case from $\kappa$ values computed from 50 Monte Carlo trials; we show results here for an average of 1 count per sinogram bin.

estimates from each sinogram for each activity level were calculated using the three different estimation methods. The mean and variances of these $\kappa$ values for each activity level were computed and compared. For each activity level, we also computed the mean values of the sinograms and used these to compute the true $\kappa$ values.

Fig. 8 shows a transaxial profile of the means of the $\kappa$ values over the Monte Carlo trials at approximately $40 \mathrm{~K}$ counts (1 count/sinogram bin). Note that $\kappa$ values outside the support of the object have no importance. The MPI improves considerably over the DPI, but GELT gives the estimate of the $\kappa$ values with the lowest bias.

Fig. 9 shows the Monte Carlo standard deviations in $\kappa$ estimates using the three methods. While the MPI exhibits smaller variance than GELT, it also has larger bias compared to GELT (as seen in Fig. 8), so that the overall mean squared error for GELT is significantly lower than that for the MPI. Figs. 8 and 9 also clearly demonstrate that the DPI method performs very poorly in both bias and standard deviation. These results are for a mean sinogram count of 1 . As the mean activity is increased, differences between the MPI and GELT methods are reduced, with the latter consistently better in terms of mean squared error. 


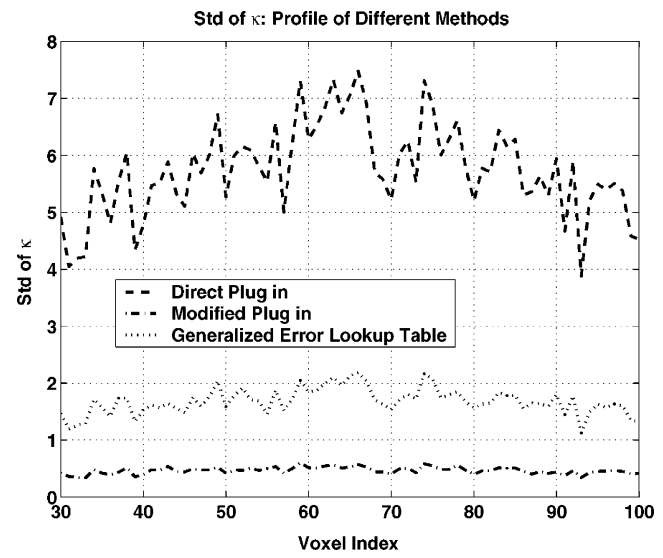

Fig. 9. The Monte Carlo standard deviations of the $\kappa$ estimates for the data in Fig. 8.

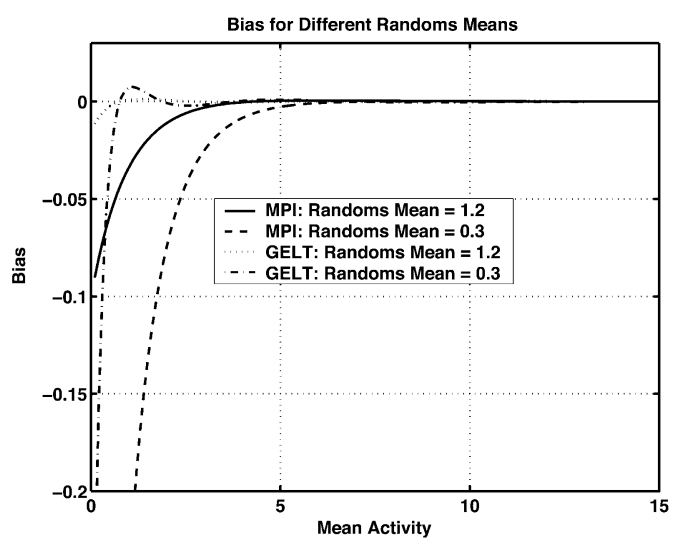

Fig. 10. Bias of $1 /(\bar{y}+2 \bar{r})$ estimates for MPI and GELT $(w=1 / 11904$, $\alpha=5$, and $\beta=2$ ) for two different randoms means (0.3 and 1.2) over a range of mean activity values $(0-15)$. GELT outperforms MPI over the entire range of typical randoms fractions (10\%-30\%).

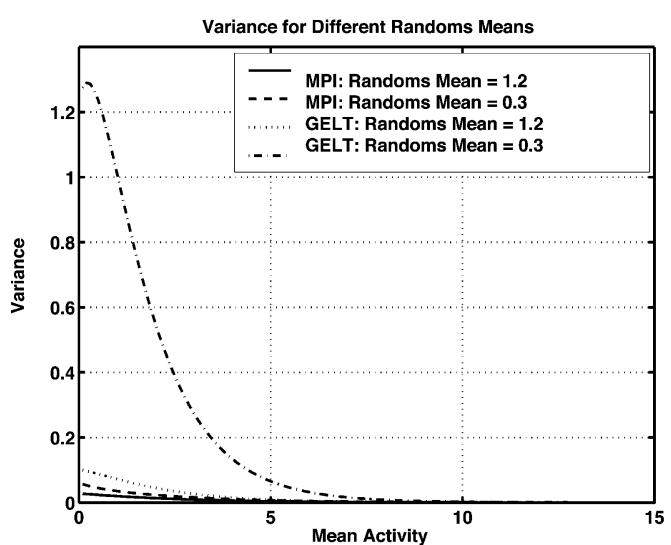

Fig. 11. Variance of $1 /(\bar{y}+2 \bar{r})$ estimates for MPI and GELT $(\alpha=5$, and $\beta=2$ ) for two different randoms means (0.3 and 1.2) over a range of mean activity values $(0-15)$. MPI has lower variance over all mean activity values but the lower bias in the GELT method results in more accurate $\kappa$ estimates.

\section{Estimation Accuracies for Randoms Precorrected Data}

The accuracy of estimates of $1 /(\bar{y}+2 \bar{r})$ are dependent on the randoms rate for randoms precorrected data. We, therefore, show the bias and standard deviation of the GELT, as well as the MPI method, for two randoms rates, .3 and 1.2., in Figs. 10

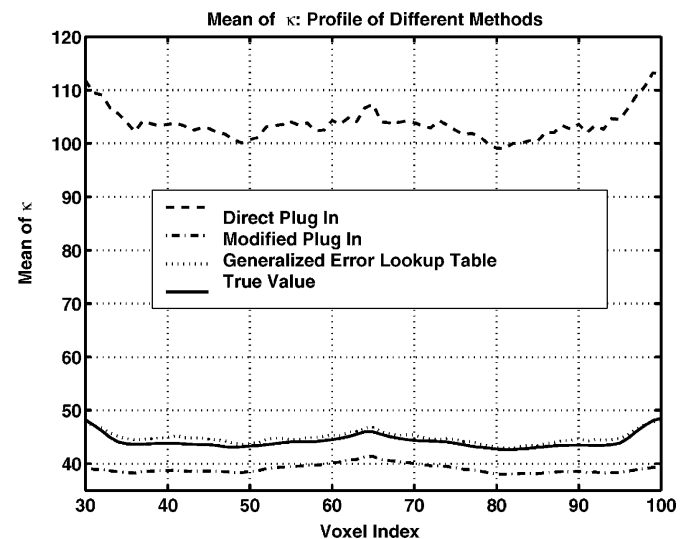

Fig. 12. The mean profile of $\kappa$ estimates from 50 Monte Carlo trials for a line through the center of the field of view of the scanner under the shifted Poisson model (mean trues: 1 count/sinogram bin; mean randoms: 0.3 counts/sinogram bin).

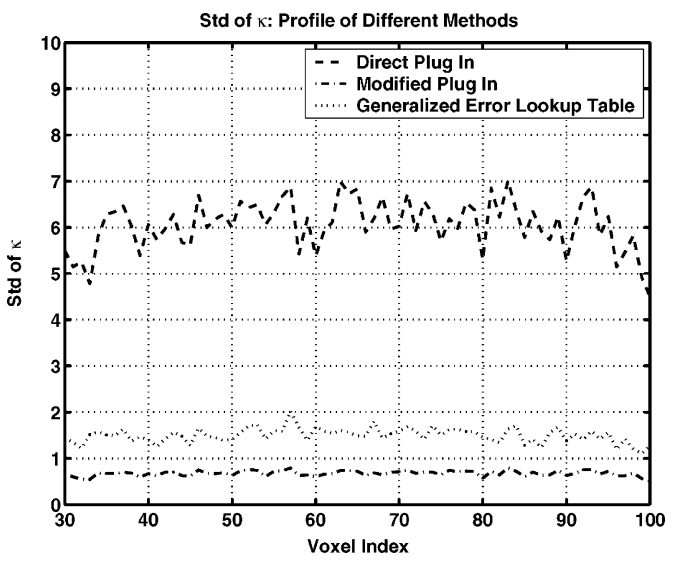

Fig. 13. The Monte Carlo standard deviations for the data in Fig. 12.

and 11 , respectively. In both cases, bias and variance for the DPI method were considerably worse and are not shown here. For both randoms rates, the GELT shows lower bias and higher variance than the MPI at the same randoms rate. The effect on the estimated FIM is shown in Figs. 12 and 13 for a mean trues rate of 1 count per sinogram element and a mean randoms rate of 0.3. As with the uncorrected Poisson case, the improvement in bias of the GELT more than outweighs the effect of increased variance compared to the MPI so that the mean squared errors for the FIM estimates using GELT are significantly lower than those using the MPI.

\section{Application to Uniform Resolution Recovery in PENALIZED ML RECONSTRUCTION}

\section{A. Uniform Resolution in Penalized ML Reconstructions}

Images reconstructed using a penalized ML objective function under the Poisson model maximize the objective function formed by the log-likelihood plus a penalty or regularizing term

$$
\hat{\mathbf{x}}(\mathbf{y})=\underset{\mathbf{x} \geq \mathbf{0}}{\operatorname{argmax}} \sum_{i=1}^{N} y_{i} \log [\mathbf{P x}]_{i}-[\mathbf{P x}]_{i}-\beta R(\mathbf{x})
$$

where $R(\mathbf{x})$ is the penalty and $\beta$ is the regularization or hyperparameter controlling the resolution-noise trade-off in reconstructed images. 
Given such an implicitly defined estimator, Fessler and Rogers [1] defined the LIR at a given voxel $j$ as the change in the mean reconstructed image in response to a small perturbation at that voxel

$$
l^{j}(\mathbf{x})=\lim _{\delta \rightarrow 0} \frac{\hat{\mathbf{x}}\left(\overline{\mathbf{y}}\left(\mathbf{x}+\delta \mathbf{e}^{j}\right)\right)-\hat{\mathbf{x}}(\overline{\mathbf{y}}(\mathbf{x}))}{\delta}
$$

where $\hat{\mathbf{x}}$ denotes the estimated image and $\overline{\mathbf{y}}$ is the measurement mean vector.

The LIR for the objective function (23) has the following approximate form that includes the FIM, F:

$$
l^{j}(\mathbf{x}) \approx[\mathbf{F}+\beta \mathbf{R}(\mathbf{x})]^{-1} \mathbf{F e}_{j}
$$

where $\mathbf{R}(\mathbf{x})$ is the second derivative matrix of the penalty at $\mathrm{X}$. In this paper, we consider only quadratic penalties so that $\mathbf{R}(\mathbf{x})=\mathbf{R}$. A more accurate expression for the LIR is given in [5, Eqns. (22) - (24)], however, this form is rather complex to work with, and the approximation in (25) has been been shown to give reasonably accurate results [2], [3], [17].

The $j$ th element of the LIR, also known as the local CRC, is a measure that can be used to characterize the resolution of a reconstructed image. Qi and Leahy [3], [17] gave the following approximation to the relation between the local CRC at voxel $j$ and the smoothing parameter $\beta$ :

$$
\operatorname{crc}_{j}=\frac{1}{N} \sum_{i=0}^{N-1} \frac{\lambda_{i}(j)}{\lambda_{i}(j)+\beta \kappa_{j}^{-2} \mu_{i}}
$$

where $\mu_{i}$ is the three-dimensional (3-D) Fourier transform of the central column of the second derivative of the penalty function, and $\lambda_{i}(j)$ is the 3-D Fourier transform of the approximation of the central column of the block-Toeplitz matrix formed from the $j$ th column of $D\left\{v_{j}\right\}^{-1} \mathbf{P}^{T} \mathbf{P} D\left\{v_{j}\right\}^{-1}$ with $v_{j}^{2}$ the $(j, j)$ th element of $\mathbf{P}^{T} \mathbf{P}$.

The LIR and, therefore, the CRC depends explicitly on the FIM. By spatially modifying the smoothing parameter $\beta$ so that $\beta \kappa_{j}^{-2}$ is constant over all voxels, we can achieve approximate invariance in CRC and, hence, approximate invariance in resolution. In particular, we use the following data-dependent penalty [1]:

$$
R(\mathbf{x})=\sum_{j} \sum_{j^{\prime} \in \mathcal{N}_{j}} w_{j j^{\prime}} \hat{\kappa}_{j} \hat{\kappa}_{j^{\prime}}\left(x_{j}-x_{j^{\prime}}\right)^{2}
$$

where $w_{j j^{\prime}}$ is the reciprocal Euclidean distance between voxels $j$ and $j^{\prime}$, and $\hat{\kappa}_{j}$ denotes the estimate for $\kappa_{j}$. Using the methods described in this paper we can apply this idea of spatially varying smoothing even for low count data using an estimate of the $\kappa$ 's based on the GELT method described above.

We note that more accurate corrections for nonuniform resolution, which take into account the spatially variant response of the scanner itself, have been proposed [5], [17]. However, our purpose here was to demonstrate the specific effects of more accurate estimation of the FIM and we have not included these factors here.

\section{B. Resolution Uniformity}

We selected 11 points of interest on the Hoffman brain phantom for resolution calculations that are shown by dark squares in Fig. 7. The resolution measurements given in this paper are the full-width at half-maximum (FWHM) values of

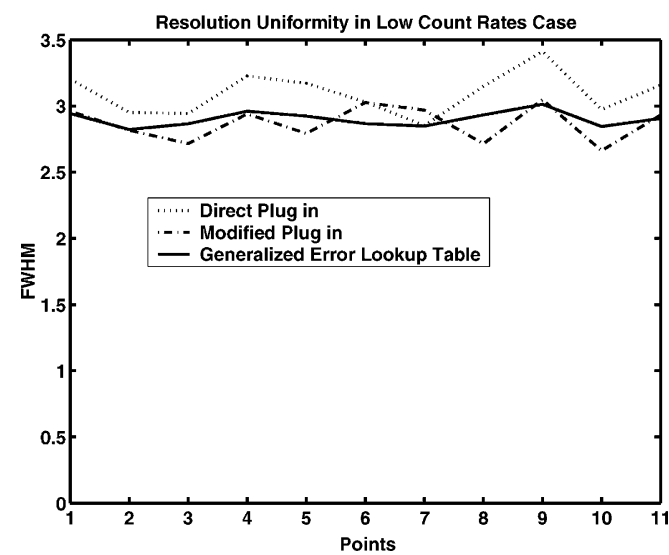

Fig. 14. Resolution at 11 points in the Hoffman brain phantom slice. Even at low counts, the GELT method still gives high spatial uniformity.

the LIR computed using Monte Carlo simulations in which we performed 100 reconstructions using the Hoffman phantom shown in Fig. 7 and performed another 100 reconstructions with the value at the point of interest perturbed by $\delta$. The difference between the mean reconstructions in both cases divided by $\delta$ gave us an approximation for the LIR. We repeated this process for all 11 points shown in Fig. 7. Although FWHM and CRC are different measures of resolution, they are highly correlated since high CRC values will lead to small FWHM values and vice versa.

The same set of noisy sinograms used to compute $\kappa$ were also used to compute and compare resolution uniformity obtained from different methods. We reconstructed the data using the MAP reconstruction method in [16] using spatially variant smoothing parameters computed using each of the three FIM estimation methods (DPI, MPI, GELT).

Fig. 14 shows the uniformity of resolution at different points when mean activity is 1 count/sinogram element. The figure indicates that the proposed method can achieve superior resolution uniformity compared to the other two methods at low count rates. The vector of parameters $\mathbf{c}=\left[C_{0}, \ldots, C_{5}\right]^{T}$ for GELT

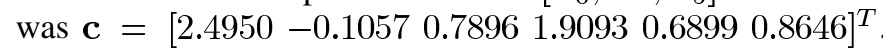
The mean and standard deviations of the FWHM values for MPI were 2.8710 and 0.1350 , respectively, whereas those for GELT were 2.9028 and 0.0584 , indicating more than a factor of 2 reduction in the standard deviations of FWHM values with GELT.

\section{CONCLUSION}

We have presented a new method for optimally estimating the FIM under ordinary Poisson and shifted Poisson models. Previous FIM estimation methods were highly biased at low counts and caused significant artifacts in applications that used the biased FIM estimates. Our minimum generalized error estimator trades off reduced bias for increased variance in the estimation of reciprocal means to optimally estimate the FIM. These improved FIM estimates can be applied for very low count data to the range of applications outlined in our introduction.

The GELT method can be readily incorporated into any statistical image reconstruction algorithm. A gamma distribution can be fit to the empirical density of the data using MATLAB's gamfit function. The $\mathbf{Q}, \mathbf{b}$ and $d$, which appear in the standard 
quadratic form, can be computed to arbitrary accuracy by increasing the number of terms used in approximating the infinite summations in (13) or (22) and the resulting convex, quadratic cost function can be maximized using any convex optimization algorithm.

The computation of the look-up table $(N=5)$ for the ordinary Poisson case took less than 5 min using MATLAB on a Pentium III 900-MHz machine. This is approximately the same time it takes to compute one forward or back projection (for ECAT HR+) on the same machine. Therefore, the use of GELT has a minimal effect on the overall image reconstruction time. An alternative would be to precompute the constants for the GELT off-line based on representative data, in which case there would be no added run-time for using this approach.

We expect GELT to be particularly useful for FIM estimation in studies that typically have low counts, such as whole body scans or dynamic PET studies with many short frames. As such an application, we demonstrated how uniform resolution could be achieved with low count Poisson data in a single-ring scanner.

\section{REFERENCES}

[1] J. A. Fessler and W. L. Rogers, "Spatial resolution properties of penalized-likelihood image reconstruction methods: Spatial-invariant tomographs," IEEE Trans. Image Processing, pp. 1346-1358, Sept. 1996.

[2] J. A. Fessler, "Mean and variance of implicitly defined biased estimators (Such as penalized maximum likelihood): Applications to tomography," IEEE Trans. Image Processing, vol. 5, pp. 493-506, Mar. 1996.

[3] J. Qi and R. M. Leahy, "A theoretical study of the contrast recovery and variance of MAP reconstructions from PET data," IEEE Trans. Med. Imag., vol. 18, pp. 293-305, Apr. 1999.

[4] J. W. Stayman and J. A. Fessler, "Regularization for uniform spatial resolution properties in penalized-likelihood image reconstruction," IEEE Trans. Med. Imag., vol. 19, pp. 601-615, June 2000.
[5] _ - "Compensation for nonuniform resolution using penalized-likelihood reconstruction in space-variant imaging systems," IEEE Trans. Med. Imag., vol. 23, pp. 269-284, Mar. 2004.

[6] - "Fast methods for approximation of resolution and covariance for SPECT," in Proc. IEEE Nuclear Science Symp./Medical Imaging Conf., Nov. 2002.

[7] P. Bonetto, J. Qi, and R. M. Leahy, "Covariance approximation for fast and accurate computation of channelized hotelling observer statistics," IEEE Trans. Nucl. Sci., vol. 47, pp. 1567-1572, Aug. 2000.

[8] H. H. Barrett, J. L. Denny, R. F. Wagner, and K. J. Myers, "Objective assessment of image quality. II. Fisher information, fourier crosstalk and figures of merit for task performance," J. Opt. Soc. Amer. A, vol. 12, no. 5, May 1995.

[9] M. F. Kijewski, S. P. Muller, and S. C. Moore, "Nonuniform collimator sensitivity: Improved precision for quantitative SPECT," J. Nucl. Med., vol. 38, no. 1, pp. 151-156, Jan. 1997.

[10] M. F. Kijewski, S. C. Moore, and H. Jadvar, "Nonuniform collimator sensitivity: Improved precision for quantitative SPECT," IEEE Trans. Nucl. Sci., vol. 48, pp. 734-738, June 2001.

[11] J. Qi and R. H. Huesman, "Theoretical study of lesion detectability of MAP reconstruction using computer observers," IEEE Trans. Med. Imag., vol. 20, pp. 815-822, Aug. 2001.

[12] J. Qi, "Optimization of PET system design for lesion detection," IEEE Trans. Nucl. Sci., vol. 48, pp. 1470-1476, August 2001.

[13] M. Yavuz and J. A. Fessler, "Statistical image reconstruction methods for randoms-precorrected PET scans," Med. Imag. Anal., vol. 2, no. 4, pp. 369-378, 1998.

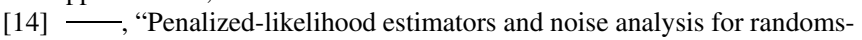
precorrected PET transmission scans," IEEE Trans. Med. Imag., vol. 18, pp. 665-674, Aug. 1999.

[15] S. Ahn and J. A. Fessler, "Statistical emission image reconstruction for randoms-precorrected PET scans using negative sinogram values," in Proc. IEEE Nuclear Science Symp./Medical Imaging Conf., 2003.

[16] J. Qi, R. M. Leahy, S. R. Cherry, A. Chatziioannou, and T. H. Farquhar, "High resolution $3 \mathrm{D}$ Bayesian image reconstruction using the microPET small-animal scanner," Phys. Med. Biol., vol. 43, no. 4, pp. 1001-1013, 1998.

[17] J. Qi and R. Leahy, "Resolution and noise properties of MAP reconstructions in fully 3D PET," IEEE Trans. Med. Imag., vol. 19, pp. 493-506, May 2000.

[18] E. Hoffman, P. Cutler, W. Didby, and J. Mazziotta, "3D phantom to simulate cerebral blood flow and metabolic images for PET," IEEE Trans. Nucl. Sci., vol. 37, pp. 616-620, Apr. 1990. 